\title{
Amelioration of bleomycin-induced pulmonary fibrosis via TGF- $\beta$-induced Smad and non-Smad signaling pathways in galectin-9-deficient mice and fibroblast cells
}

Yu-An Hsu' ${ }^{1 \dagger}$, Ching-Yao Chang ${ }^{2 \dagger}$, Joung-Liang Lan ${ }^{3,4,5}$, Ju-Pi Li ${ }^{3,4}$, Hui-Ju Lin ${ }^{1,6}$, Chih-Sheng Chen ${ }^{7,8}$, Lei Wan ${ }^{1,2,9^{*}}$ and Fu-Tong Liu ${ }^{10,11^{*}}$

\begin{abstract}
Background: Galectin-9 is a $\beta$-galactoside-binding protein with two carbohydrate recognition domains. Recent studies have revealed that galectin-9 regulates cellular biological reactions and plays a pivotal role in fibrosis. The aim of this study was to determine the role of galectin-9 in the pathogenesis of bleomycin-induced systemic sclerosis (SSC).

Methods: Human galectin-9 levels in the serum of patients with SSC and mouse sera galectin-9 levels were measured by a Bio-Plex immunoassay and enzyme-linked immunosorbent assay. Lung fibrosis was induced using bleomycin in galectin-9 wild-type and knockout mice. The effects of galectin-9 on the fibrosis markers and signaling molecules in the mouse lung tissues and primary lung fibroblast cells were assessed with western blotting and quantitative polymerase chain reaction.

Results: Galectin-9 levels in the serum were significantly higher (9-fold) in patients compared to those of healthy individuals. Galectin-9 deficiency in mice prominently ameliorated epithelial proliferation, collagen I accumulation, and $a$-smooth muscle actin expression. In addition, the galectin-9 knockout mice showed reduced protein expression levels of fibrosis markers such as Smad2/3, connective tissue growth factor, and endothelin-1. Differences between the wild-type and knockout groups were also observed in the AKT, mitogen-activated protein kinase, and c-Jun N-terminal kinase signaling pathways. Galectin-9 deficiency decreased the signal activation induced by transforming growth factor-beta in mouse primary fibroblasts, which plays a critical role in fibroblast activation and aberrant catabolism of the extracellular matrix.
\end{abstract}

Conclusions: Our findings suggest that lack of galectin-9 protects against bleomycin-induced SSc. Moreover, galectin-9 might be involved in regulating the progression of fibrosis in multiple pathways.

Keywords: Galectin-9, Bleomycin, TGF- $\beta$, Fibrosis, Systemic sclerosis

\footnotetext{
* Correspondence: lei.joseph@gmail.com; ftliu@ibms.sinica.edu.tw; fliu@ucdavis.edu

${ }^{\dagger}$ Yu-An Hsu and Ching-Yao Chang contributed equally to this work.

${ }^{1}$ School of Chinese Medicine, China Medical University, No. 91, Hsueh-Shih

Road, Taichung 40402, Taiwan

${ }^{10}$ Institute of Biomedical Sciences, Academia Sinica, Taipei 11529, Taiwan

Full list of author information is available at the end of the article
}

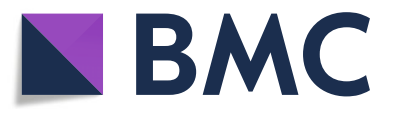

(c) The Author(s). 2020 Open Access This article is distributed under the terms of the Creative Commons Attribution 4.0 International License (http://creativecommons.org/licenses/by/4.0/), which permits unrestricted use, distribution, and reproduction in any medium, provided you give appropriate credit to the original author(s) and the source, provide a link to the Creative Commons license, and indicate if changes were made. The Creative Commons Public Domain Dedication waiver (http://creativecommons.org/publicdomain/zero/1.0/) applies to the data made available in this article, unless otherwise stated. 


\section{Background}

Systemic sclerosis ( $\mathrm{SSc}$ ) is a chronic systemic connective tissue disease that exhibits characteristics such as essential vasculopathy; fibrosis in the skin, subcutaneous tissue, muscles, and internal organs (e.g., alimentary tract, lungs, heart, kidney, central nervous system); and immunologic activation $[1,2]$. The pathophysiology of SSc is unknown, and there are no effective therapies for the disease. Recent studies of the multifaceted etiopathogenesis for the whole disease or organ-specific SSc have revealed numerous molecular targets for potential therapeutic interventions [3]. For many years, activation of fibroblasts was considered to result in excess extracellular matrix deposition in the pathogenesis of SSc. However, recent evidence suggests that the activation of fibroblasts is in fact orchestrated by other cells.

Several potent profibrotic genes were found to be upregulated in SSc, including transforming growth factor beta (TGF- $\beta$ ), interleukin-4 (IL-4), platelet-derived growth factor, monocyte chemoattractant protein-1, and connective tissue growth factor (CTGF) [4]. TGF- $\beta$ is expressed in activating fibroblasts and elevates the synthesis of collagen, which is encoded by the COL1A1 gene. TGF- $\beta$ also increases proteoglycan synthesis and inhibits extracellular matrix degradation by decreasing matrix metalloproteinase (MMP) synthesis and enhancing tissue inhibitor of MMP expression [5]. TGF- $\beta$ binds to its receptor TGF $\beta$ RI to activate its transducing signal into the nucleus via Smad2 and Smad3 phosphorylation. Smad6 and Smad7 are inhibitory Smads that mediate negative feedback by inhibiting TGF- $\beta$ signaling via forming a complex with Smurf E3 ubiquitin ligase. Moreover, disrupting the functions of Smad3 and Smad7 in SSc reduces the degree of fibrosis [6]. Endothelin-1 (ET-1) and CTGF are produced by endothelial cells and fibroblasts in the early and late phases of SSc. ET-1 is a vasoconstrictor that can stimulate collagen synthesis and inhibit MMP expression, leading to vasculopathy in SSc. CTGF was also observed to be overexpressed in SSc by TGF- $\beta$-activated fibroblasts to stimulate collagen production [7, 8].

Galectin-9 is a $36-\mathrm{kDa} \beta$-D-galactoside-binding protein comprised of two distinct carbohydrate recognition domains connected by a linker peptide in the $\mathrm{N}$-and $\mathrm{C}$ termini [9]. The galectin family is thought to regulate cell homeostasis and inflammation. Previous studies demonstrated that galectin-9 is distributed among tissues and induces various biological reactions such as cell aggregation, adhesion, chemoattraction, activation, and apoptosis [10]. Galectin-9 regulates the Th1/Th17 cell ratio to balance the immune response, thus playing a role in inflammatory diseases, and regulates $\mathrm{T}$-cell immunity in chronic hepatitis $C$ virus infection $[11,12]$. In addition, galectin-9 expression was reported to be significantly elevated in the serum and lesional skin of patients with SSc, it was also considered to contribute to the Th immune balance in the lesional skin of SSc [13].

However, the role of galectin-9 in the pulmonary fibrosis of SSc remains unknown. In the present study, the expression level of galectin-9 in the lungs of patients with fibrosis was evaluated. Moreover, the effect of galectin-9 on fibrotic markers of mouse lung fibroblast cells and lung tissues was assessed in vitro and in vivo.

\section{Material and methods}

\section{Patients and galectin-9 detection}

Serum samples were obtained from 26 patients with SSc and 26 healthy controls. These samples were frozen at $80{ }^{\circ} \mathrm{C}$ until analysis. Human galectin-9 levels were measured at the Inflammation Core Facility of the Institute of Biomedical Sciences, Academia Sinica, using their multiplex assay to measure cytokine/chemokine levels in patient sera. The level of the mediator was determined by a Bio-Plex 200 analyzer, which is a dual-laser, flowbased, sorting and detection platform. Additional information on the system can be found at the manufacturer's website (https://www.ibms.sinica.edu.tw/ inflammation_core_facility/page/s-a.html).

The mouse galectin-9 levels were detected by an enzyme-linked immunoassay kit from Elabscience (Houston, TX, USA) according to the manufacturer instructions.

\section{Mice}

The Lgals9 knockout (KO) mice (strain B6(FVB)Lgals $9^{\text {tm1.1cfg }} /$ Mmucd) were established by Dr. Jim Paulson. The commercial source can purchase from Mutant Mouse Resource and Research Center (MMRRC). Detailed genetic information of Lgals9 $\mathrm{KO}$ mice can be found on the CFG functional glycomics gateway site (http://www.functionalglycomics.org/static/consortium/ resources/resourcecoref.shtml) or MMRRC (Citation ID: MMRRC_031952-UCD). C57BL/6 J mice were suggested as the wild-type (WT) controls per the MMRRC recommendation. Ten-to-twelve-week-old male mice were used for the experiments. The mice were obtained and bred in Taiwan National Laboratory Animal Center and National Applied Research Laboratories (NARLabs, Taipei, Taiwan), and housed according to the Principles of Laboratory Animal Care. The procedures for animal care and handling were approved by the Animal Committee of China Medical University. There were six to eight mice per group.

\section{Cell culture}

Primary lung fibroblast cells were cultured from 8-weekold mice. In brief, the lungs of Lgals9 WT and KO mice were removed, added to Dulbecco's modified Eagle's 
medium containing $10 \%$ fetal bovine serum, and triturated using trypsin. Dissociated cells were then plated in $10-\mathrm{cm}$ dishes and cultured for 1-2 weeks. After culturing, $3 \times 10^{5}$ cells were seeded into 6 -cm dishes and incubated with $40,20,10,5$, and $1 \mathrm{ng} / \mathrm{ml}$ recombinant murine TGF- $\beta$ (PeproTech, Rocky Hill, NJ, USA) for 24 $h$, since TGF- $\beta$ is a well-established primary mediator driving fibrogenesis [14]. The cells were then collected for RNA and protein extraction.

\section{Bleomycin-induced murine model of lung fibrosis and respiratory resistance}

Bleomycin has been found to induce DNA strand breaks and oxidative stress, resulting in direct injury to the cell [15]. Subsequently, cell death occurs through either necrosis or apoptosis, accompanied by inflammation and fibrosis. To induce pulmonary fibrosis, bleomycin (Cayman Chemical, Ann Arbor, MI, USA) was applied intratracheally at $80 \mu \mathrm{g}$ in a total volume of $20 \mu \mathrm{l}$ to the mice twice a week, and the mice were sacrificed 4 weeks later. This dose was previously confirmed in mice for intratracheal delivery [16]. For intratracheal injection, the mice were placed in supine position on the operating field and the trachea was exposed with an otoscope. The bleomycin solution was injected into the trachea directly with a syringe through a 25 -gauge needle. The lung tissues were isolated for further analyses. Airway responsiveness was expressed using the "enhanced pause" (Penh) as a parameter of altered airway function [17]. Penh is an empirical parameter that reflects changes in the box flow waveform from both inspiration and expiration. To measure respiratory system resistance, the mice were subjected to whole-body plethysmography for Penh recording (DSI Buxco, St. Paul, MN, USA).

\section{Histopathology and immunofluorescent staining}

The lung tissues were fixed in 10\% neutral-buffered formalin and embedded in paraffin. The tissues were cut into $5-\mu \mathrm{m}$ sections and placed on slides, followed by staining with hematoxylin and eosin $(\mathrm{H} \& \mathrm{E})$ and Masson's trichrome (Leica Biosystems, Wetzlar, Germany). For immunofluorescent staining, $16-\mu \mathrm{m}$ sections from O.C.T. (a tissue freezing medium)-embedded frozen tissues were blocked with $5 \%$ bovine serum albumin, incubated at room temperature with anti-alpha-smooth muscle actin ( $\alpha$-SMA) antibody (GeneTex, Irvine, CA, USA) overnight at $4{ }^{\circ} \mathrm{C}$, and then incubated with Texas Red-conjugated secondary antibody (GeneTex) for $1 \mathrm{~h}$ at room temperature. The nuclei were stained with DAPI for $5 \mathrm{~min}$ at room temperature, and images were acquired with a fluorescence microscope (Olympus, Tokyo, Japan).
RNA extraction and reverse transcription-quantitative polymerase chain reaction (qPCR)

Total RNA of the lung tissues and fibroblast cells was isolated using an RNeasy mini Kit (Qiagen, Hilden, Germany), and cDNA was synthesized using a HighCapacity cDNA Reverse Transcriptase Kit (Applied Biosystems, Foster City, CA, USA) according to the manufacturers' instructions. ACTA2, COL1A1, CTGF, and ET1 transcript levels were then measured by qPCR using the cDNA as a template on a StepOne Plus system (Applied Biosystems) with universal probes (Roche, Basel, Switzerland) and the specific primer pairs listed in Table 1 . The threshold cycle number $(\mathrm{Ct})$ was calculated for each gene and normalized to that of glyceraldehyde 3-phosphate dehydrogenase $(G A P D H)$. The $\triangle \mathrm{Ct}$ values for each gene are presented as relative fold-induction.

\section{Western blotting analysis}

The cells were washed twice with cold phosphatebuffered saline and lysed with RIPA lysis buffer $(50 \mathrm{mM}$ Tris- $\mathrm{HCl} \mathrm{pH} 7.4,150 \mathrm{mM} \mathrm{NaCl}, 1 \% \mathrm{NP} 40,0.25 \% \mathrm{Na}-$ deoxycholate, $1 \mathrm{mM}$ PMSF) supplemented with a protease and phosphatase inhibitor cocktail (Roche). The protein concentrations of the cell lysis extracts were measured using the Bradford protein assay (Bio-Rad, Hercules, CA, USA) and equalized with the extraction reagent. Equal amounts of the proteins were loaded and subjected to sodium dodecyl sulfate-polyacrylamide gel electrophoresis, transferred onto $0.2-\mu \mathrm{m}$ polyvinylidene fluoride membranes (Millipore, Billerica, MA, USA), and stained with appropriate antibodies (CTGF, ET-1, aSMA, and beta-actin: GeneTex, Irvine, CA, USA); Smad2, phospho-Smad2, Smad3, phospho-Smad3, Smad2/3, p-AKT, AKT, p- mitogen-activated protein kinase (MAPK), MAPK, p-c-Jun N-terminal kinase (JNK), and JNK: Cell Signaling Technologies, Danvers, MA, USA). The membranes were incubated with a 1: 5000-10,000 dilution of horseradish peroxidaseconjugated anti-mouse or anti-rabbit secondary antibody

\begin{tabular}{|c|c|c|c|}
\hline Gene & Primer & Sequence $5^{\prime}-3^{\prime}$ & Probe no. \\
\hline ACTA2 & Forward & 5'- CCAGCACCATGAAGATCAAG - 3' & 58 \\
\hline ACTA2 & Reverse & 5'- TCCACATCTGCTGGAAGGTA - 3' & \\
\hline COL1A1 & Forward & 5'- CATGTTCAGCTTTGTGGACCT - 3' & 15 \\
\hline COL1A1 & Reverse & 5'- GCAGCTGACTTCAGGGATGT - 3' & \\
\hline CTGF & Forward & 5'- TGACCTGGAGGAAAACATTAAGA - 3' & 71 \\
\hline CTGF & Reverse & 5'- AGCCCTGTATGTCTTCACACTG - 3' & \\
\hline ET-1 & Forward & 5'- TCCTTGATGGACAAGGAGTGT - 3' & 29 \\
\hline ET-1 & Reverse & 5'- CCCAATCCATACGGTACGAC - 3' & \\
\hline GAPDH & Forward & 5'- GCCAAAAGGGTCATCATCTC - 3' & 29 \\
\hline GAPDH & Reverse & 5'- CACACCCATCACAAACATGG -3' & \\
\hline
\end{tabular}


(Cell Signaling) at room temperature for $2 \mathrm{~h}$. Membranes were developed using the ECL system (ThermoFisher Scientific, Waltham, MA, USA) according to the manufacturer's protocols. The reaction was visualized by chemiluminescence using an ImageQuant LAS4000 mini system (GE Healthcare, Little Chalfont, UK). Band intensity was quantified with ImageJ software (National Institutes of Health, Bethesda, MD, USA) and protein levels were normalized by beta-actin. In the graphs, the relative value compared with the control group is expressed as mean \pm SD in arbitrary units.

\section{Statistical analysis}

The data of galectin- 9 expression in human and mouse sera were analyzed by the Mann-Whitney test and Student's $t$-tests. Pearson's coefficient analysis was used to analyze the correlation between the forced vital capacity (FVC) or diffusion capacity (DLCO) and galectin-9 expression level in the sera of SSc patients. The other data were analyzed using Student's $t$-tests. A $p$ value $<0.05$ was considered statistically significant.

\section{Results}

\section{Galectin-9 levels are increased in the serum of SSC} patients

To investigate the contribution of galectin-9 to SSc, the concentration of galectin- 9 in the serum was determined by bio-plex immunoassay. Galectin- 9 levels were significantly higher (9-fold) in patients with SSc compared to those of healthy controls $(p<0.0001$, Fig. 1). This result indicates that galectin-9 might be involved in the pathogenesis of SSc. We also examined the association of serum galectin-9 levels with clinical pulmonary function tests,

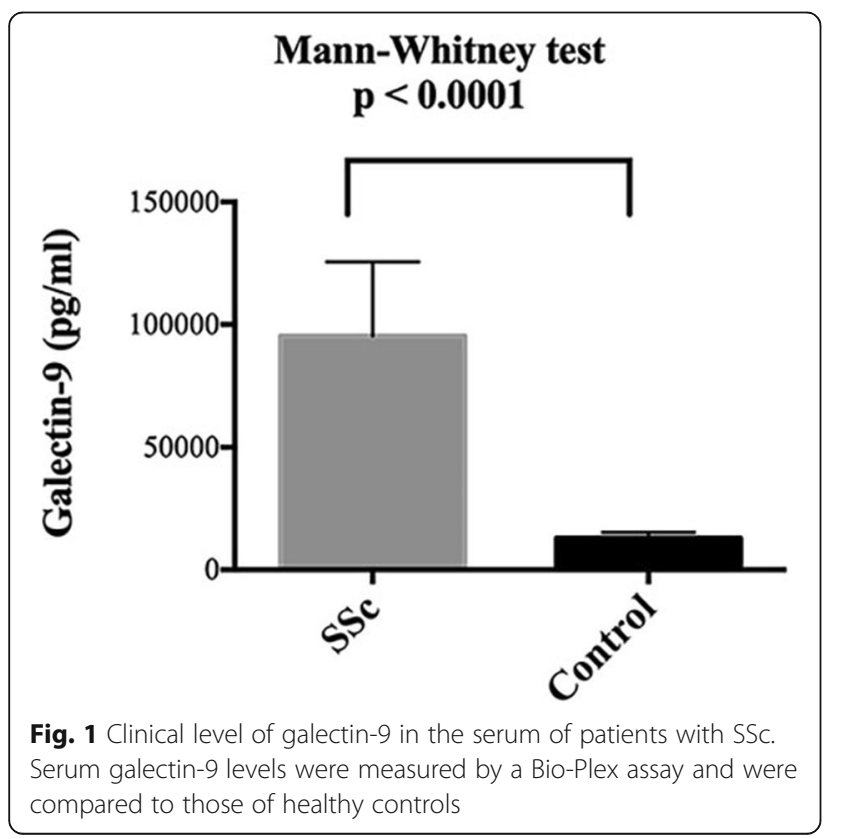

including FVC and DLCO. We used Pearson's correlation analysis to analyze the relationship between FVC or DLCO and galectin-9 expression levels in the serum of SSc patients, demonstrating a strong negative correlation with FVC but a weaker correlation with DLCO: FVC vs. galectin-9: $r=-0.737$; DLCO vs. galectin-9: $r=-0.446$ (mean FVC: $71.9 \pm 14.3 \%$, mean DLCO: $52.2 \pm 19 \%$, mean galectin-9 level: $31153 \pm 18,832 \mathrm{pg} / \mathrm{ml}$ ).

\section{Galectin-9 deficiency attenuated bleomycin-induced lung fibrosis}

Fibrosis is the major characteristic of SSc, and bleomycin has been shown to cause pulmonary fibrosis [18]. Therefore, we next investigated the effect of galectin- 9 on pulmonary fibrosis induced by bleomycin in mice. Bleomycin was administered into the lung via the intratracheal route at $80 \mu \mathrm{g}$ twice weekly for 4 weeks to induce fibrosis. Before sacrifice, the mice were subjected to whole-body plethysmography to detect the level of Penh. Galectin-9 KO mice showed lower levels of Penh $(p<0.041$ Fig. 2a) than those of WT mice. Thus, respiratory inflammation was ameliorated in mice with galectin- 9 deficiency. In addition, the serum galectin-9 level was significantly increased in bleomycin-treated WT mice compared with that of saline-treated WT mice $(p=0.0248$, Fig. $2 \mathrm{~b})$. In the lung tissues, bleomycin induced fibrosis development to a greater extent in WT mice than in galectin-9 KO mice according to $\mathrm{H} \& \mathrm{E}$ staining (Fig. 2c, upper). Collagen fiber production was also higher in WT tissues according to Masson trichrome staining (Fig. 2c, middle). Moreover, the intensity of $\alpha$ SMA staining, which plays an important role in fibrogenesis, was higher in WT than in galectin-9 KO mouse lung tissues (Fig. 2c, below). Consistently, qPCR using the WT tissues revealed significantly higher mRNA expression levels of ACTA2, COL1A1, and CTGF (Fig. 2d). Furthermore, the levels of the fibrotic proteins Smad2/3, CTGF, and ET-1 were determined by western blotting. The CTGF expression level in galectin-9 WT mice was significantly higher $(p<0.05)$ than that of the galectin-9 KO mouse tissues. Although galectin-9 KO tissues exhibited slightly lower expression levels of Smad2/3 and ET-1, the differences were not significantly significant between the two groups (Fig. 2e). Collectively, these results indicate that galectin- 9 expression is involved in fibrosis progression through TGF- $\beta$-activated ACTA2, CTGF, and ET-1. In contrast, fibrosis was improved when galectin-9 was deficient.

\section{Lack of galectin-9 decreased the progression of fibrosis by TGF- $\beta$}

To examine the difference in galectin-9 WT and KO mice in the development of TGF- $\beta$-activated fibrosis, we examined primary fibroblast cells from both types of 


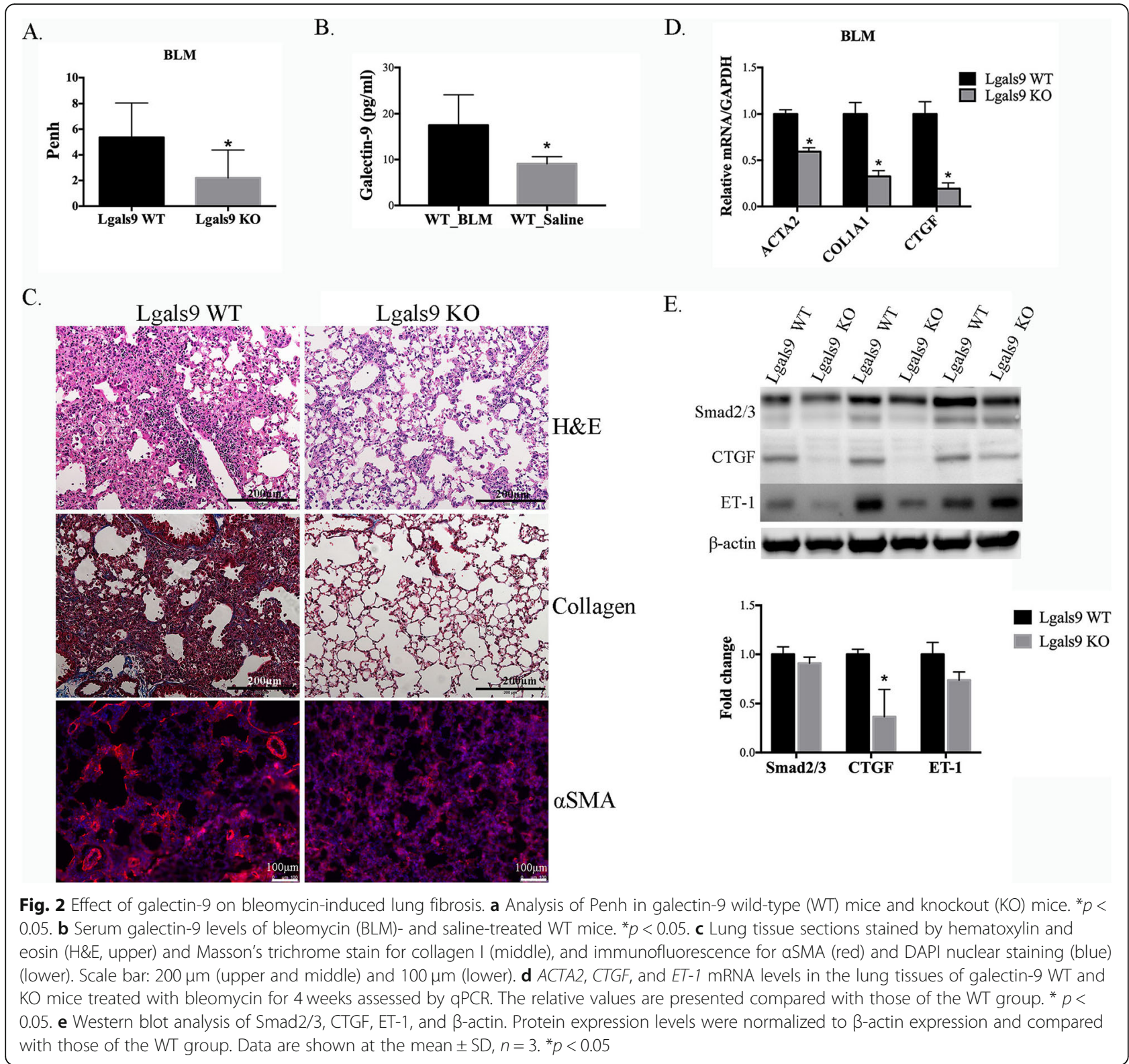

mice. TGF- $\beta$ induced $\alpha$ SMA expression in a dosedependent manner in WT fibroblast cells. In addition, the fold-change in TGF- $\beta$-induced $\alpha$ SMA protein expression was markedly higher in WT cells than in $\mathrm{KO}$ cells respectively compared with that of control cells not treated with TGF- $\beta$ (Fig. $3 \mathrm{a}$ and b). Similar effects on COL1A1, CTGF, and ET-1 were observed by qPCR (Fig. 3c). Finally, we evaluated the Smaddependent pathway induced by TGF- $\beta$. TGF- $\beta$ induced transcriptional regulation by phosphorylating the Smad2 and Smad3 proteins, followed by an interaction with Smad4. As shown in Fig. 3d, TGF- $\beta$ significantly induced Smad2 and Smad3 phosphorylation in WT tissues. Cells from the mice defective in galectin-9 showed a reduced response to TGF- $\beta$.
These findings indicate that lack of galectin-9 in fibroblasts suppresses TGF- $\beta$-related reactions.

\section{Galectin-9 deficiency affects fibrosis progression via a Smad-independent pathway in vivo and in vitro}

TGF- $\beta$ has been recognized as a central mediator of tissue fibrosis. The major mechanism involves delivery of the signal via Smad molecules through the Smad-dependent pathway. However, the MAPK/ extracellular related kinase (ERK), P38, c-JNK, nuclear factor- $\mathrm{kB}$, and phosphatidylinositol 3-kinase (PI3K) signaling pathways have also been implicated in the fibrosis responses induced by TGF- $\beta$ [19]. To evaluate the effect of galectin-9 deficiency on the Smad-independent pathways of fibrosis, the lung 


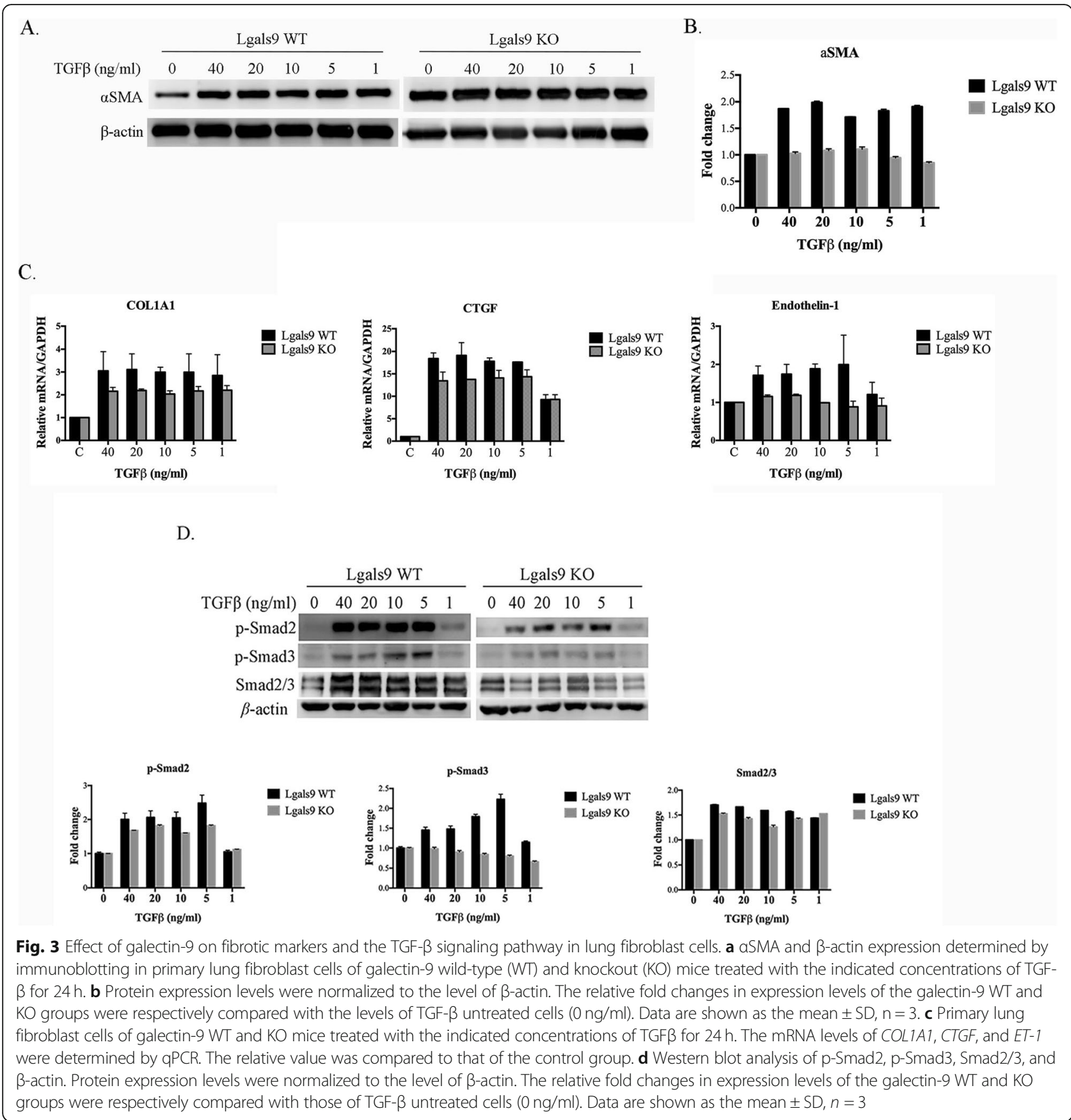

tissues of bleomycin-treated galectin-9 WT and KO mice were isolated and the proteins were extracted. The levels of phosphorylated AKT, MAPK, and JNK proteins were higher in galectin-9 WT than those of KO mice (Fig. 4a). Consistent results were observed in mouse primary lung fibroblast cells; the change in phosphate AKT and MAPK by TGF- $\beta$ showed a greater increase following administration of galectin9 (Fig. 4b). These findings indicate that galectin-9 promotes signaling pathways that cause fibrosis via TGF- $\beta$.

\section{Discussion}

SSc is a chronic systemic autoimmune disease characterized as a multisystemic connective tissue disease caused by inflammation and fibrosis on the skin or internal organs [1]. The major pathogenic factors responsible for the various clinical features are vascular injury, fibrosis, and immune activation [20]. Studies have also indicated that inflammatory cytokines induce endothelial cell injury leading to vascular damage [21]. Expression of these cytokines can cause fibrosis by inducing excess extracellular matrix synthesis and collagen accumulation. The 


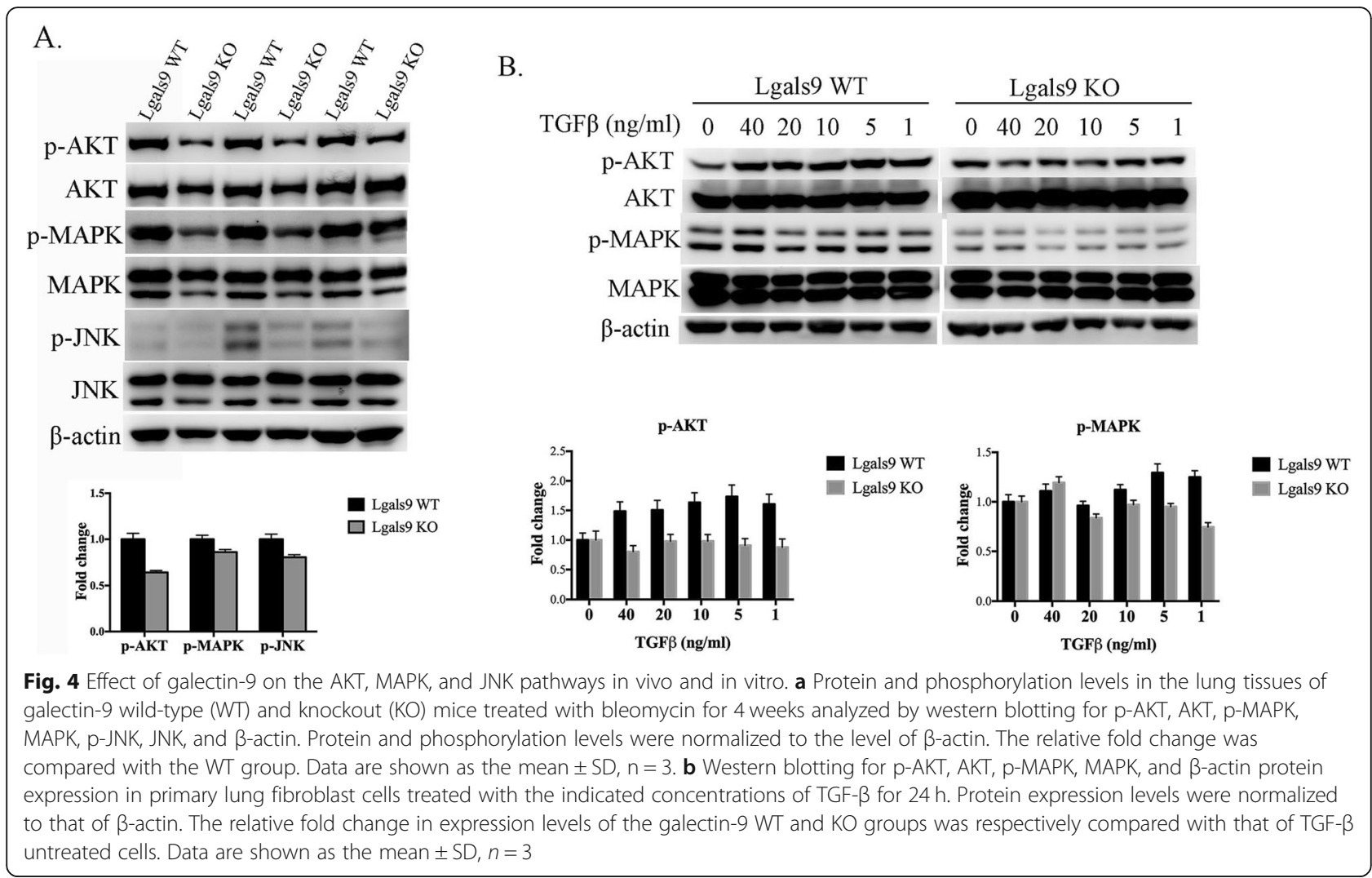

vascular injury is mediated by ET-1 and TGF- $\beta$ activation, which contribute to fibroblast activation and myofibroblast transdifferentiation [22]. Myofibroblasts expressing $\alpha \mathrm{SMA}$ are the primary moderators of fibrosis caused by the excess deposition of extracellular matrix [23]. TGF- $\beta$ induces $\alpha$ SMA expression via the Smad signaling pathways, and can induce ET-1 and CTGF production through Smad-dependent or Smad-independent signaling pathways, including the MAPK/ERK, TAK1/ JNK, and PI3K/AKT pathways, which are also activated by TGF- $\beta$ [19].

However, the factors contributing to the initiation of the pathogenesis of SSc remain unclear. The administration of bleomycin is a widely used method to induce pulmonary fibrosis in animal models. Bleomycin induces DNA strand breaks and oxidative stress to directly injure the cell. Subsequently, cell death occurs through either necrosis or apoptosis with consequent development of inflammation and fibrosis [16]. We found that the levels of fibrotic markers such as $\alpha \mathrm{SMA}, \mathrm{CTGF}$, and collagen were significantly higher in the bleomycin-induced lung fibrosis model than controls, suggesting a suitable model for assessing the role of galectin-9 in the lung fibrosis accompanying SSc.

Galectin-9 is a $\beta$-galactoside lectin with two carbohydrate recognition domains at the $\mathrm{N}$ - and $\mathrm{C}$-termini connected by a linker peptide. Galectin- 9 is encoded by
LGALS9, which is widely distributed among tissues, and is predominantly expressed by activated endothelial cells, interferon (IFN)-stimulated fibroblasts, and innate or adaptive immune cells [24]. Galectin-9 is highly expressed in the liver and circulation in patients with chronic liver diseases, and higher serum galectin-9 levels are related to liver fibrosis progression [25]. The clinical association of serum galectin-9 levels with SSc has also been investigated in patients [13]. Similar results were observed in our study, with higher expression of galectin-9 detected in the serum of patients with SSc. Thus, galectin-9 might be useful as a biomarker of fibrosis in SSc.

TGF- $\beta$ is known to induce fibroblast growth and collagen synthesis. Enhanced TGF- $\beta$ signaling has been observed in SSc fibroblasts in vivo and in vitro [26]. Upon TGF- $\beta$ binding to the TGF- $\beta$ receptors T $\beta R I$ and T $\beta R I$, the receptors are phosphorylated and transiently associate with Smads (Smad2 and Smad3). Receptor-activated Smads are phosphorylated and then form a heterooligomeric complex with Smad4 for translocation to the nucleus to induce target gene transcription [27]. TGF- $\beta$ regulates expression of the $\alpha$ SMA gene (ACTA2) via Smad3 activation in myofibroblast differentiation [22]. Moreover, increased Smad2 and Smad3 phosphorylation by TGF- $\beta$ was observed in scleroderma fibroblasts [8]. TGF- $\beta$ is mainly derived by the peripheral naïve $\mathrm{T}$ cells 
that differentiate into Foxp3 ${ }^{+}$iTreg cells. Smad3 enhances Foxp3 expression, and activation of Smad3 and Foxp3 ensure the stable formation of iTreg cells. Previous studies showed that mice lacking galectin- 9 exhibited decreased Foxp3 expression levels, and Lgals9 ${ }^{-1-} \mathrm{T}$ cells were defective in Foxp3 expression. Galectin-9 promotes iTreg differentiation via the TGF- $\beta$-induced phosphorylation of Smad2/3, MAPK/ERK, and complex formation of Smad2/3 with Smad4 [28, 29]. These data demonstrate that galectin- 9 participates in further regulation through both Smad-dependent and -independent pathways. In line with the results of the present study, normal expression of galectin-9 results in high activation of Smad2/3 and significant fold-induction of ACTA2, COL1A1, CTGF, and ET-1 expression. Galectin-9 deficiency reduced the production of these fibrotic molecules. TGF- $\beta$ activated the Smad-independent MAPK/ ERK, TAK1/JNK, and PI3K/AKT pathways to mediate tissue fibrosis. We also found that TGF- $\beta$ induced higher levels of phosphorylation of AKT, MAPK, and JNK in both the lung tissues and lung fibroblast cells of WT mice. In contrast, lack of galectin-9 ameliorated the progression of fibrosis by TGF- $\beta$.

In terms of immunity, $\mathrm{CD} 4^{+} \mathrm{T}$ cell activation and infiltration in the skin and internal organs occurs in the early phase of SSc. Activated T cells, B cells, and nonspecific inflammatory cells infiltrate various tissues to cause damage to the fibroblasts and endothelial cells by inducing several mediators [30]. The balance between Th1 and Th2 cytokines is altered in tissue injury. T cells polarized toward the Th2 pattern, which secrete abundant IL-4, IL-5, and IL-13, contribute to formation of a pro-fibrotic environment [31]. In contrast, the Th1 cytokine IFNY is associated with anti-fibrotic effects. Lower levels of IFN $\gamma$ in the blood and a deficiency of IFNY production in peripheral mononuclear cells and bronchoalveolar lavage cells have been investigated in patients with SSc. Studies of Th2/Th17-skewed immune polarization in SSc indicated that IL-33 production induced the skin-localized transdifferentiation of Tregs into Th2-like cells [32]. The immunomodulatory effect of galectin-9 has been studied based on its interaction with the glycoprotein ligand TIM-3. TIM-3 is highly expressed on Th1 cells and by activated $\mathrm{CD} 4^{+}$cells in humans [33]. In addition, activated $\mathrm{CD}^{+} \mathrm{T}$ cells secreted IFN $\gamma$, IL-17, IL-2, and IL-6, but not IL-10, IL-4, or tumor necrosis factor- $\alpha$. In mice, galectin- 9 also mediated the decrease in Th1 and Th17 cell infiltration, which was associated with the downregulation of CXCL9, CXCL10, and CCL20 expression [11]. Elevated galectin-9 expression was also observed in SSc dermal fibroblasts in vivo and in vitro. Importantly, galectin-9 overproduction can suppress IFN $\gamma$ expression by $\mathrm{CD}_{4}^{+}$ $\mathrm{T}$ cells in $\mathrm{Fli1}^{+/-}$dermal fibroblasts. Bleomycin-induced skin fibrosis was attenuated by galectin-9 deficiency and increased IFN $\gamma$ production $[11,13]$. These findings were similar with our present results, suggesting that the loss of galectin-9 significantly reduces fibrosis and plays a role in the balance of Th1/Th2 immunity.

\section{Conclusion}

We investigated the role of galectin-9 expression in the serum of patients with SSc. We found increased expression levels of collagen and $\alpha \mathrm{SMA}$ in lung sections of mice induced to develop lung fibrosis with bleomycin as an SSc animal model. In addition, higher mRNA expression levels of ACTA2, COL1A1, and CTGF were observed in the bleomycin-treated lung specimens of mice expressing normal levels of galectin-9 compared to those with galectin-9 deficiency. Similar results for the protein expression of Smad2/3, CTGF, and ET-1 were detected under galectin- 9 deficiency. Activation of TGF- $\beta$ signaling was shown to up-regulate the expression of downstream fibrotic markers in fibroblast cells expressing galectin-9. Moreover, the MAPK/ERK, TAK1/JNK, and PI3K/AKT signaling pathways were influenced by the presence of galectin-9 in lung tissues and fibroblast cells. These results suggest that galectin- 9 acts as a potent mediator of fibrosis progression.

In conclusion, our findings suggest an important role for galectin- 9 as a mediator of the TGF- $\beta$-induced progression of lung fibroblast cells into fibrosis. Data from the bleomycin-induced lung fibrosis model showed consistent results. We also demonstrated that galectin-9 promotes fibrosis development via the entire TGF- $\beta$ signaling pathway. Therefore, galectin- 9 is a potential biomarker that may also serve as a novel target for therapeutic intervention in SSc.

\section{Acknowledgements \\ We thank the patients and control individuals who participate in this study. The authors thank Editage for help with English editing.}

\section{Authors' contributions}

$L W, J L L$ and FTL conceived and designed the study. YAH and JPL performed the experiments. JLL and FTL provided the specimen. CYC and CSC analyzed the experiments data. YAH and CYC wrote the paper. LW reviewed and edited the manuscript. All authors read and approved the final manuscript.

\section{Funding}

This study was supported in part by the Ministry of Science and Technology, Taiwan, R.O.C. (MOST103-2314-B-039-035-MY3 and MOST105-2628-B-039008-MY3), China Medical University Hospital, Taichung, Taiwan (DMR-108211), and China Medical University, Taichung, Taiwan (CMU107-ASIA-23). The sponsor or funding organization had no role in the design or conduct of this research.

\section{Availability of data and materials}

The data that support the findings of this study are available from the corresponding author upon reasonable request.

Ethics approval and consent to participate

The SSc patients' sera were obtained from Dr. Lan and this study was approved by the Institutional Review Board (IRB) of China Medical University 
Hospital, Taichung, Taiwan (cmuh105-rec3-005). The usage of all clinica specimens was anonymous.

All animal studies were according to protocols approved by Laboratory Animal Committee of China medical University (Approval No.2018-226)

\section{Consent for publication}

Not applicable.

\section{Competing interests}

The authors declare that the research was conducted in the absence of any commercial or financial relationships that could be construed as a potential conflict of interest.

\section{Author details}

${ }^{1}$ School of Chinese Medicine, China Medical University, No. 91, Hsueh-Shih Road, Taichung 40402, Taiwan. ${ }^{2}$ Department of Biotechnology, Asia University, Taichung 40402, Taiwan. ${ }^{3}$ Rheumatology Research Center, China Medical University Hospital, Taichung 40402, Taiwan. ${ }^{4}$ School of Medicine, China Medical University, Taichung 40402, Taiwan. ${ }^{5}$ Division of Immunology and Rheumatology, Department of Internal Medicine, China Medical University Hospital, Taichung 40402, Taiwan. ${ }^{6}$ Department of Ophthalmology, China Medical University Hospital, Taichung 40402, Taiwan. ${ }^{7}$ Division of Chinese Traumatology, China Medical University Hospital, Taichung 40402, Taiwan. ${ }^{8}$ Division of Chinese Medicine, Asia University Hospital, Taichung 40402, Taiwan. ${ }^{9}$ Department of Gynecology, China Medical University Hospital, Taichung 40402, Taiwan. ${ }^{10}$ Institute of Biomedical Sciences, Academia Sinica, Taipei 11529, Taiwan. ${ }^{11}$ Department of Dermatology, University of California, Davis, School of Medicine, Sacramento, CA 95816, USA.

Received: 20 July 2019 Accepted: 9 January 2020

Published online: 15 January 2020

\section{References}

1. Denton CP. Systemic sclerosis: from pathogenesis to targeted therapy. Clin Exp Rheumatol. 2015;33(4 Suppl 92):S3-7.

2. Mayes MD, Lacey JV Jr, Beebe-Dimmer J, Gillespie BW, Cooper B, Laing TJ, et al. Prevalence, incidence, survival, and disease characteristics of systemic sclerosis in a large US population. Arthritis Rheum. 2003;48(8):2246-55.

3. Sakkas LI. New developments in the pathogenesis of systemic sclerosis. Autoimmunity. 2005;38(2):113-6.

4. Sakkas LI, Platsoucas CD. Is systemic sclerosis an antigen-driven T cell disease? Arthritis Rheum. 2004;50(6):1721-33.

5. Nabel EG, Shum L, Pompili VJ, Yang ZY, San H, Shu HB, et al. Direct transfer of transforming growth factor beta 1 gene into arteries stimulates fibrocellular hyperplasia. Proc Natl Acad Sci U S A. 1993;90(22):10759-63.

6. Derynck R, Zhang Y, Feng XH. Smads: transcriptional activators of TGF-beta responses. Cell. 1998;95(6):737-40.

7. Abraham DJ, Vancheeswaran R, Dashwood MR, Rajkumar VS, Pantelides P, Xu SW, et al. Increased levels of endothelin-1 and differential endothelin type $\mathrm{a}$ and $\mathrm{B}$ receptor expression in scleroderma-associated fibrotic lung disease. Am J Pathol. 1997;151(3):831-41.

8. Dong C, Zhu S, Wang T, Yoon W, Li Z, Alvarez RJ, et al. Deficient Smad7 expression: a putative molecular defect in scleroderma. Proc Natl Acad Sci U S A. 2002;99(6):3908-13.

9. Wada J, Kanwar YS. Identification and characterization of galectin-9, a novel beta-galactoside-binding mammalian lectin. J Biol Chem. 1997;272(9):607886.

10. Bacigalupo ML, Manzi M, Rabinovich GA, Troncoso MF. Hierarchical and selective roles of galectins in hepatocarcinogenesis, liver fibrosis and inflammation of hepatocellular carcinoma. World J Gastroenterol. 2013; 19(47):8831-49.

11. Zhang Q, Luan H, Wang L, He F, Zhou H, Xu X, et al. Galectin-9 ameliorates anti-GBM glomerulonephritis by inhibiting Th1 and Th17 immune responses in mice. Am J Physiol Renal Physiol. 2014;306(8):F822-32.

12. Fujita K, Niki T, Nomura T, Oura K, Tadokoro T, Sakamoto T, et al. Correlation between serum galectin-9 levels and liver fibrosis. J Gastroenterol Hepatol. 2018;33(2):492-9.

13. Saigusa R, Asano Y, Nakamura K, Hirabayashi M, Miura S, Yamashita T, et al. Systemic sclerosis dermal fibroblasts suppress Th1 cytokine production via
Galectin-9 overproduction due to Fli1 deficiency. J Invest Dermatol. 2017; 137(9):1850-9.

14. Meng XM, Nikolic-Paterson DJ, Lan HY. TGF-beta: the master regulator of fibrosis. Nat Rev Nephrol. 2016;12(6):325-38.

15. Moore BB, Hogaboam CM. Murine models of pulmonary fibrosis. Am J Physiol Lung Cell Mol Physiol. 2008;294(2):L152-60.

16. Moore BB, Lawson WE, Oury TD, Sisson TH, Raghavendran K, Hogaboam CM. Animal models of fibrotic lung disease. Am J Respir Cell Mol Biol. 2013; 49(2):167-79.

17. Frazer DG, Reynolds JS, Jackson MC. Determining when enhanced pause (Penh) is sensitive to changes in specific airway resistance. J Toxicol Environ Health A. 2011;74(5):287-95.

18. Adamson IY, Bowden DH. The pathogenesis of bleomycin-induced pulmonary fibrosis in mice. Am J Pathol. 1974;77(2):185-97.

19. Lee CM, Park JW, Cho WK, Zhou Y, Han B, Yoon PO, et al. Modifiers of TGFbeta1 effector function as novel therapeutic targets of pulmonary fibrosis. Korean J Intern Med. 2014;29(3):281-90.

20. O'Reilly S, Hugle T, van Laar JM. T cells in systemic sclerosis: a reappraisal. Rheumatology (Oxford). 2012;51(9):1540-9.

21. Asano Y, Sato S. Vasculopathy in scleroderma. Semin Immunopathol. 2015; 37(5):489-500.

22. Hu B, Wu Z, Phan SH. Smad3 mediates transforming growth factor-betainduced alpha-smooth muscle actin expression. Am J Respir Cell Mol Biol. 2003:29(3 Pt 1):397-404.

23. Cherng $S$, Young $Y$, Ma H. Alpha-smooth muscle actin a-SMA. Am J Sci. 2008;4(4):7-9.

24. Igawa K, Satoh T, Hirashima M, Yokozeki H. Regulatory mechanisms of galectin-9 and eotaxin-3 synthesis in epidermal keratinocytes: possible involvement of galectin-9 in dermal eosinophilia of Th1-polarized skin inflammation. Allergy. 2006;61(12):1385-91.

25. Golden-Mason L, Rosen HR. Galectin-9: diverse roles in hepatic immune homeostasis and inflammation. Hepatology. 2017;66(1):271-9.

26. Ayers NB, Sun CM, Chen SY. Transforming growth factor-beta signaling in systemic sclerosis. J Biomed Res. 2018;32(1):3-12.

27. Chen F, Weinberg RA. Biochemical evidence for the autophosphorylation and transphosphorylation of transforming growth factor beta receptor kinases. Proc Natl Acad Sci U S A. 1995;92(5):1565-9.

28. Derynck R, Zhang YE. Smad-dependent and Smad-independent pathways in TGF-beta family signalling. Nature. 2003;425(6958):577-84.

29. Nagae M, Nishi N, Murata T, Usui T, Nakamura T, Wakatsuki S, et al. Structural analysis of the recognition mechanism of poly- $\mathrm{N}$ acetyllactosamine by the human galectin- $9 \mathrm{~N}$-terminal carbohydrate recognition domain. Glycobiology. 2009;19(2):112-7.

30. White B. Immunopathogenesis of systemic sclerosis. Rheum Dis Clin N Am. 1996;22(4):695-708.

31. Mavalia C, Scaletti C, Romagnani P, Carossino AM, Pignone A, Emmi L, et al. Type 2 helper T-cell predominance and high CD30 expression in systemic sclerosis. Am J Pathol. 1997;151(6):1751-8.

32. MacDonald KG, Dawson NA, Huang Q, Dunne JV, Levings MK, Broady R. Regulatory T cells produce profibrotic cytokines in the skin of patients with systemic sclerosis. J Allergy Clin Immunol. 2015;135(4):946-e9.

33. Vega-Carrascal I, Reeves EP, Niki T, Arikawa T, McNally P, O'Neill SJ, et al. Dysregulation of TIM-3-galectin-9 pathway in the cystic fibrosis airways. J Immunol. 2011;186(5):2897-909.

\section{Publisher's Note}

Springer Nature remains neutral with regard to jurisdictional claims in published maps and institutional affiliations. 